\title{
Classification of Bluffing Behavior and Affective Attitude from Prefrontal Surface Encephalogram During On-Line Game
}

\author{
Myung Hwan Yun, Joo Hwan Lee, Hyoung-joo Lee, and Sungzoon Cho \\ Department of Industrial Engineering, \\ Seoul National University, Seoul, 151-742 South Korea \\ \{mhy, leejh337, impatton, zoon\}@snu.ac.kr
}

\begin{abstract}
The purpose of this research was to detect the pattern of player's emotional change during on-line game. By defining data processing technique and analysis method for bio-physiological activity and player's bluffing behavior, the classification of affective attitudes during on-line game was attempted. Bluffing behavior displayed during the game was classified into two dimensions of emotional axis based on prefrontal surface electroencephalographic data. Classified bluffing attitudes were: (1) pleasantness/unpleasantness; and (2) honesty/bluffing. A multilayer-perception neural network was used to classify the player state into four attitude categories. Resulting classifier showed moderate performance with $67.03 \%$ pleasantness/unpleasantness classification, and $77.51 \%$ for honesty/bluffing. The classifier model developed in this study was integrated to on-line game as a form of 'emoticon' which displays facial expression of opposing player's emotional state.
\end{abstract}

\section{Introduction}

Although bio-electrical signal was known since late 1840s, forms of BCI (Brain Computer Interface), which facilitates human-system interaction using various biosignals, were introduced only in 1970s. Later on, through various researches (Hesham, 2003; Yuko, 2002; Pfurtscheller et al., 1996), basic interface functions such as controlling mouse cursors by EEG signal are being reported. On the other hand, there were substantial researches attempting to discriminate emotional state of human beings in real life situation such as fatigue, drowsiness, and general stress level using bio-physiological signals (Eoh et. al., 2005). With continuous improvement of signal measurement and digital processing technology, BCI is rapidly becoming an important option for biometric interaction. While it is far from being a realistic authentification tool, BCI is continuously expanding its application area. Brain is the primary center for the regulation and control of bodily activities, re-ceiving and interpreting bio signals, and transmitting information (Andreassi, 1995). By attaching electrodes on the scalp, the electrical activity of the brain can be recorded. Details of the EEG and its processing are out of the scope of this paper. There are numerous sources of information related to this area (Gevins et al., 1998; Wilson et al., 1999). A polygraph is an instrument that records changes in physiological processes such as heart rate, 
blood pressure, respiration and other bio-signals. The polygraph test is frequently used in practical situation. The underlying assumption of the polygraph test is that when people deviate from their normal state, they produce measurable changes in their physiological signal. A baseline for testing these physiological characteristics is established by asking various questions whose answers the investigator already knows. Deviation from the baseline for truthfulness is taken as sign of lying or bluffing (Luan K., 2005; Vance, 2001). There are three basic approaches to the logic behind affective state classification with polygraph test (shown the table1) and they are used as the underlying concept for developing the affective attitude classification model of this study.

Table 1. Three approaches to affective attitudes classification

\begin{tabular}{l|l}
\hline \multicolumn{1}{c|}{ Approaches } & \multicolumn{1}{c}{ Method } \\
\hline $\begin{array}{l}\text { The Control Question } \\
\text { Test (CQT). }\end{array}$ & $\begin{array}{l}\text { This test compares the physiological response to relevant questions } \\
\text { about the crime with the response to questions relating to prior } \\
\text { misdeeds. "This test is often used to determine whether certain } \\
\text { criminal suspects should be classified as uninvolved in the crime" }\end{array}$ \\
\hline $\begin{array}{l}\text { The Directed Lie (or } \\
\text { bluffing) Test (DLT) }\end{array}$ & $\begin{array}{l}\text { This test tries to detect lying by comparing physiological responses } \\
\text { when the subject is told to deliberately bluffing to responses when } \\
\text { they tell or act the truth. }\end{array}$ \\
\hline $\begin{array}{l}\text { The Guilty Knowl- } \\
\text { edge Test (GKT) }\end{array}$ & $\begin{array}{l}\text { This test compares physiological responses to multiple-choice type } \\
\text { questions about the crime, one choice of which contains informa- } \\
\text { tion only the investigators and the criminal would know about. }\end{array}$ \\
\hline
\end{tabular}

\section{Experiment}

Fifteen students (10 males, 5 females, mean age, 25.8, S.D. \pm 3.51 ) who have fair amount of experience in on-line game participated for data collection. The experimental equipment used for signal measurement and analysis was developed specifically for this study. The equipment consists of a hair band with EEG sensors, heart rate sensors and signal transmission unit. For the EEG measurement, four prefrontal channels (two forehead channels, one Reference, and one Ground) were used. The band pass filter was butter-worth with $4-46 \mathrm{~Hz}$ range with the gain of 4,700. For the heart rate measurement, a direct reflex sensor (exposing the skin to direct-current infra-red beam and extracting blood pulse signal from the IR reflexion data, band-pass range, 2 to $10 \mathrm{~Hz}$, gain 6,000) was attached inside the EEG hair band. Figure 1 illustrates the experimental settings. Based on the polygraph paradigm, game situations and player reactions were classified as pleasant (advantageous) and unpleasant (disadvantageous) situations.

Player reaction was classified to two separate state; aggressive and conservative. Table 2 also shows the scheme of affective attitude classification used in this study; honesty and bluffing. Figure 2 is the task analyses chart which is used to branch and bound the possibility of various outcomes from the attitudes classification structure in Table2. 


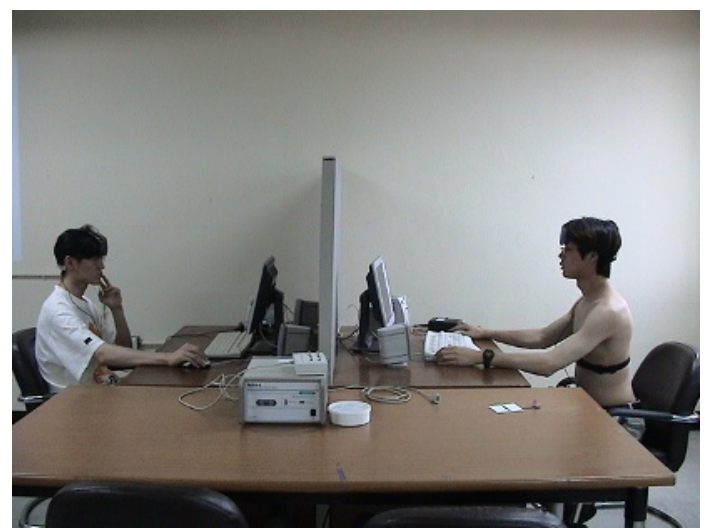

Fig. 1. Experimental set up (players, EEG/ECG input devices (hair band), signal transmission unit, and heart rate monitors, attached to subject's chest area)

Table 2. Classification of player bluffing and affective attitudes

\begin{tabular}{c|c|c}
\hline & $\begin{array}{c}\text { Pleasantness } \\
\text { (Advantageous situation) }\end{array}$ & $\begin{array}{c}\text { Unpleasantness } \\
\text { (Disadvantageous situation) }\end{array}$ \\
\hline Aggressive betting & Honesty & Bluffing \\
\hline Conservative betting & Bluffing & Honesty \\
\hline
\end{tabular}

Four type of EEG band, alpha, beta, theta, and delta were filtered by FFT analysis. Changes in affective attitudes was detected through an EEG signal coming from right frontal region, Fp2 (based on 10-20 International System, Waldstein et al, 2000, Kimbrell et al., 1999). After the measurement equipments were attached, a session of the on-line game was conducted with fifteen matches (10 minutes of session were used for the measurement of baseline EEG). After 10 minutes rest, 30 sets of on-line game were conducted (including 10 minutes rest between each 15 sets). Saved video files were later shown to each subject for the post-hoc evaluation. First, input variable of EEG signal was selected. The raw data collected through experiment was divided at 0.33 second interval and classified into four frequency bands $(\alpha, \beta, \delta, \theta)$. The following is the input vector that was created by calculating the RMS value of each wave.

$$
\begin{gathered}
\text { RMS value: } \chi_{\mathrm{i}}=\sqrt{1 /\left|P_{i}\right| \sum_{i} P_{i}^{2}} \quad(\mathrm{i}=\alpha, \beta, \delta, \theta) \\
\text { Input vector: } \mathrm{X}=\left[\mathrm{x}_{\alpha}, \mathrm{x}_{\beta}, \mathrm{x}_{\delta}, \mathrm{x}_{\theta}\right]^{\mathrm{T}}
\end{gathered}
$$

Output variable was evaluated as the follows; Output variable1 $\left(\mathrm{y}_{1}\right)$ : pleasantness (advantage) or unpleasantness (disadvantage).

$$
\begin{aligned}
& \mathrm{y}_{1}=1: \text { When the participants feel advantageous } \\
& =0: \text { When the participants feel disadvantageous }
\end{aligned}
$$


Output variable2 $\left(\mathrm{y}_{2}\right)$ : honesty or bluffing

$$
\begin{aligned}
\mathrm{y}_{2} & =1: \text { When the participants play bluffing } \\
& =0: \text { When the participants play honestly }
\end{aligned}
$$

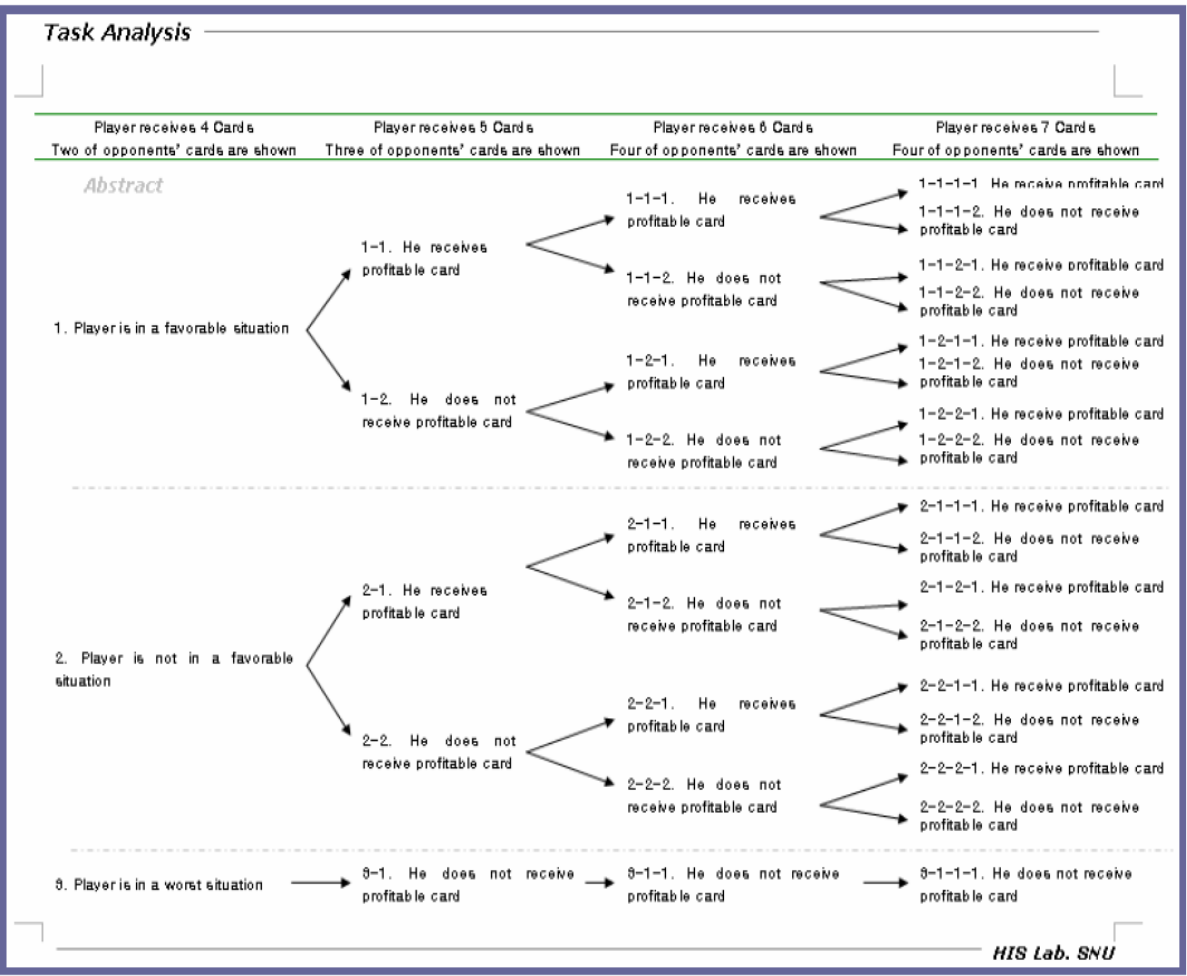

Fig. 2. Task structures and game plan used for attitudes classification (on-line poker game)

Table 3. Data distribution classified by output variables

\begin{tabular}{c|c|c|c|c}
\hline \multirow{2}{*}{} & \multicolumn{2}{c|}{ Output variable 1 (y1) } & \multirow{2}{*}{ Total } \\
\cline { 3 - 4 } \multicolumn{2}{c|}{} & Pleasantness (1) & Unpleasantness (0) & \\
\hline \multirow{2}{*}{$\begin{array}{c}\text { Output } \\
\text { variable } 2 \\
(y 2)\end{array}$} & Bluffing(1) & $\begin{array}{c}135 \\
(2.48 \%)\end{array}$ & $\begin{array}{c}1,159 \\
(21.31 \%)\end{array}$ & $\begin{array}{c}1,293 \\
(23.80 \%)\end{array}$ \\
\cline { 3 - 5 } & Honesty(0) & $\begin{array}{c}3,192 \\
(58.75 \%)\end{array}$ & $\begin{array}{c}948 \\
(17.45 \%)\end{array}$ & $\begin{array}{c}4,140 \\
(76.20 \%)\end{array}$ \\
\hline \multicolumn{2}{c|}{ Total } & $\begin{array}{c}3,327 \\
(61.24 \%)\end{array}$ & $\begin{array}{c}2,106 \\
(38.76 \%)\end{array}$ & 5,433 \\
\hline
\end{tabular}


The EEG data was collected through fifteen subjects and the data for subject evaluation was also collected. In general, change of emotion was relatively more noticeable in the latter half of the trial than in the early stage in the EEG data. Therefore, only EEG data at the time of the sixth and seventh card (latter half) was selected and the EEG pattern was developed from selecting the data when the subject evaluation score was over 9 point (very highly confident). A total 5,433 set of data was created from four input variable and two output variables. The data distribution classified by output variable is as shown in Table 3.

\section{Results}

\subsection{Analysis of Various Classification Model}

Model Analysis, which compares each candidate model by accuracy, generates the optimal EEG analysis model. For selecting the model, 1,800 data among whole data were used. The criterion of comparing each model was classification accuracy. Linear Discriminant Analysis (LDA) spent less training time than the other data mining models. So, it can be suitable for an on-line game in real time. But LDA produced higher training and test errors than others. RBF kernel SVM was used for model analysis (Burges, 1998). For RBF kernel SVM, 2 parameters should be specified: the kernel width, ' $r$ ' and the penalty for misclassification, 'cost'. The highest classification accuracy was obtained at $r=1$ and $\cos t=30$. ANN (Artificial Neural Network) was also used for model analysis. A back propagation multi-layer perceptron (1 hidden layer) using the Levenberg-Marquadt algorithm was employed. Hidden layer activation functions were as follows. The highest classification accuracy was obtained with 10 hidden nodes. The results are summarized in Table 4. Bagging Neural Network (BNN) was carried out. When 10 networks with 10 hidden nodes were combined, the highest classification performance was obtained (Breiman, 1996).

\subsection{Selection of the Best Model}

Among the 5,433 data, 1,800 data were extracted for candidate model selection. To select the suitable model, criteria such as; (1) possibility of real time analysis; (2) high classification accuracy; and (3) easiness to develop the program was considered. Table 5 is a summary of experimental results. ANN turns out to be the most suitable model. LDA spent the shortest training time and was very easy to apply but its accuracy was too low. SVM was more accurate than LDA but is impossible for real time analysis and spent relatively longer time for training. ANN achieved the highest accuracy, spent relatively shorter training time and was easy to apply. Eventually, ANN was selected for final EEG analysis model.

\subsection{EEG Index Model}

Using the basis of the ANN model, the final EEG index was developed. EEG index is as follows:

Discrimination of pleasantness/unpleasantness: Score $1=\operatorname{round}(y 1 \times 4+1)$

Discrimination of honesty/bluffing: $\quad$ Score $2=\operatorname{round}(y 2 \times 99+1)$ 
Table 4. The analysis results using ANN

(Unit: \%)

\begin{tabular}{c|c|c|c|c|c|c|c}
\hline \multirow{2}{*}{} & \multicolumn{7}{|c}{ Hidden nodes } \\
\cline { 2 - 8 } & 3 & 5 & 10 & 15 & 20 & 25 & 30 \\
\hline Output variable 1 & 70.10 & 72.30 & 72.95 & 64.73 & 72.30 & 72.79 & 72.62 \\
\hline Output variable 2 & 70.64 & 67.69 & 67.58 & 71.77 & 68.64 & 67.48 & 67.62 \\
\hline Combination & 55.34 & 54.75 & 56.15 & 5.019 & 56.11 & 55.51 & 55.65 \\
\hline
\end{tabular}

Table 5. Experimental results and features of each model

(Unit: \%)

\begin{tabular}{c|c|c|c|c}
\hline & LDA & SVM & ANN & BNN \\
\hline Output variable 1 & 50.60 & 62.38 & 67.03 & 62.23 \\
\hline Output variable 2 & 54.72 & 75.83 & 77.51 & 76.40 \\
\hline Combination & 36.61 & 55.79 & 58.92 & 58.37 \\
\hline Accuracy & too bad & good & good & good \\
\hline Training time & very good & good & good & normal \\
\hline Easiness of application & very good & normal & good & bad \\
\hline
\end{tabular}

In pleasantness/unpleasantness index, index score range was set to 1 to 5 . Higher score means higher level of pleasantness displayed by the gamer. In honesty/bluffing classification index, index score range was set to 1 to 100 such that higher score means higher probability of bluffing behavior. Eventually, the two kind of index developed from the model was programmed as a form of 'emoticon' so that facial expression of opposing gamer can be displayed together with the cards being played in the game. Using the blood pulse rate obtained from the IR sensor, conversion to Heart Rate (HR) was conducted. The variation of HR during the game was used for detecting the difference between pleasantness and unpleasantness. As the result, when HR was increased, pleasantness/unpleasantness score increased. Since the relationship between pleasantness/unpleasantness index and HR was not statistically significant, HR was used only as the weight value of pleasantness/unpleasantness index.

\section{Conclusion}

The purpose of this research was to classify the affective attitudes during on-line game using a EEG-based data processing technology. This study also suggested an index approach to quantify the player's behavior through the psychophysiological dimension of pleasantness/unpleasantness and honesty/bluffing. Since the approach used a real-time, continuously updating strategy, the classification scheme will be improved continuously as the game progresses. Although resulting classifier model showed moderate performance with $67.03 \%$ pleasantness/unpleasantness classification, and $77.51 \%$ for honesty/bluffing classification, it is higher than expected level considering that the model will be used in real-time, continuously updated situation. Together with the classification model, an on-line game with EEG measurement was 
also developed and implemented in this study. The EEG of game players were measured, transferred, and displayed to the other player during an on-line game in the form of an 'emoticon' displaying a various facial expression according to pleasantness/unpleasantness and honesty/bluffing scores calculated from the classifier.

\section{References}

1. Andreassi, J.L.: Psychophysiology: Human Behavior \& Physiological Response, 3rd ed., Lawrence Erlbaum Associates, New Jersey (1995)

2. Bishop, C.M.: Neural Network for Pattern Recognition. Oxford University Press (1995)

3. Breiman L.: Bagging predictors, Machine Learning, 24(2) (1996) 123-140

4. Burges, C.J.C.: A tutorial on support vector machines for pattern recognition, Data Mining and Knowledge Discovery, 2 (1998) 121-167

5. Eoh, H.J., Chung, M.K. and Kim, S. H.: Electroencephalographic study of drowsiness in simulated driving with sleep deprivation, International Journal of Industrial Ergonomics, 35(4) (2005) 307-320

6. Gevins, A., Smith, M.E., Leong, H., Mcevoy, L., Whitfield, S., Du, R., and Rush, G.: Monitoring working memory load during computer-based tasks with EEG pattern recognition methods, Human Factors, 40(1) (1998) 79-91

7. Hesham Sheikh, Dennis J. McFarland, William A. Sarnacki and Jonathan R. Wolpaw: Electroencephalographic (EEG)-based communication- EEG control versus system performance in humans, Neuroscience Letters, 345(2) (2003), 89-92

8. Kimbrell T.A., Mark S. George, Priti I. Parekh, Terence A. Ketter, Daniel M. Podell, Aimee L. Danielson, Jennifer D. Repella, Brenda E. Benson, Mark W. Willis, Peter Herscovitch and Robert M. Post: Regional brain activity during transient self-induced anxiety and anger in healthy adults, Biological Psychiatry, 46(4) (1999) 454-465

9. Luan, K.: Neural correlates of telling lies: A functional magnetic resonance imaging study at 4 Tesla, Academic Radiology, 12(2) (2005) 164-172

10. Pfurtscheller G., G.R. Miller, C. Guger: Direct control of a robot by electrical signals from the brain, proceeding EMBEC '99, Part 2, (1999) 1354-1355

11. Vance V.: A quantitative review of the guilty knowledge test, Journal of applied psychology, 86(4) (2001) 674-683

12. Waldstein, S.R., Kop W.J., Schmidt, L.A.: Frontal electro cortical and cardiovascular reactivity during happiness and anger, Biological Psychology, 55(1) (2000) 3-23

13. Wilson, G.F., Swain, C.R., and Ullsperger, P.: EEG Power Changes during a Multiple Level Memory Retention Task, International Journal of Psychophysiology, 32 (1999) $107-$ 118

14. Yuko Ishiwaka, Hiroshi Yokoi and Yukinori Kakazu: EEG on-line analysis for autonomous adaptive interface, International Congress Series, 1232 (2002) 271-275

15. Yun, M.H.: Development of an adaptive computer game interface based on biophysiological signal processing technique, Ministry of Science and Technology, South Korea (2000) (unpublished research report, in Korean) 